Z. klin. Chem. u. klin. Biochem.

10. Jg. 1972 , S. $156-159$

\title{
Eine gaschromatographische Bestimmung quaternärer Ammoniumverbindungen
}

\author{
Von H.-J. VIDIC, H. Dross und H. Kewriz \\ Institut für Klinische Pharmakologie, Klinikum Steglitz der Freien Universität Berlin
}

(Eingegangen am 19. Juli/26. August 1971)

Es wurde eine gaschromatographische Methode zur Bestimmung von quaternären Ammoniumverbindungen, z. B. Cholin und dessen Ester, Neostigmin, d-Tubocurarin, Methylcurarin und Scopolamin-butylbromid (Buscopan) ausgearbeitet. Die genannten Verbindungen lassen sich noch in Mengen von 0,1 nMol erfassen. Zur Vorreinigung einiger quaternärer Ammoniumverbindungen wird deren Abtrennung in Form der Jodkomplexe vorgeschlagen. Besonders Scopolamin-butylbromid, d-Tubocurarin, Methylcuratin und Neostigmin lassen sich auf diese Weise mit geringem Aufwand sehr weitgehend von den meisten lipophilen und hydrophilen Begleitstoffen aus biologischen Extrakten abtrennen.

\section{A gas chromatographic determination of quaterriary ammonium compounds}

A gas chromatographic method was developed for the determination of quaternary ammonium compounds, e. g. choline and its esters, neostigmine, d-tubocurarine, methylcurarine and scopolamine-butyl bromide (buscopan), in quantities down to $0.1 \mathrm{nmole}$. It is recommended that some quaternary ammonium compounds be subjected to a preliminary purification by the separation of their iodide complexes. This treatment is especially suitable for separating scopolamine-butyl bromide, d-tubocurarine, methylcurarine and neostigmine from most of the lipophilic and hydrophilic contaminants found in biological extracts.

Die Bestimmungsmethoden für quaternäre Ammoniumverbindungen (1-6), die als Arzneimittel verwendet werden, sind für den quantitativen Nachweis in Harn, Blut oder Organen nicht ausreichend empfindlich, zu ungenau oder zu kompliziert. Für die Analyse des körpereigenen Acetylcholins werden noch immer die Kontraktionen des Froschmuskels oder des Blutegels herangezogen, falls nicht sogar die sehr aufwendige Béstimmung am Blutdruck der eviscerierten Katze vorgenommen wird. Diese biologischen Verfahren haben mancherlei prinzipielle Nachteile, die chemisch-physikalischen Meßmethoden nicht anhaften. Deshalb wurde eine Bestimmung ausgearbeitet, die auf der gaschromatographischen Trennung beruht.

Ausgangspunkt unserer Untersuchungen waren Arbeiten von SztLagyi, Schmidt und Green (1), die sich der Pyrolyse bedienten, um eine Alkylgruppe quantitativ abzuspalten. Die Abspaltung einer Alkylgruppe läßt sich auch durch Einwirkung von Natriumthiophenolat erreichen, wie von I. HANIN und Mitarbeitern (2) beschrieben wurde.

Nach Desalkylierung von Cholinestern sind beide Reaktionsprodukte flüchtig und können gaschromatographisch aufgetrennt werden, während bei anderen Ammoniumverbindungen nur das entstandene Alkylhalogenid gaschromatographisch nachgewiesen werden kann.

Die Reaktion verläuft nach:

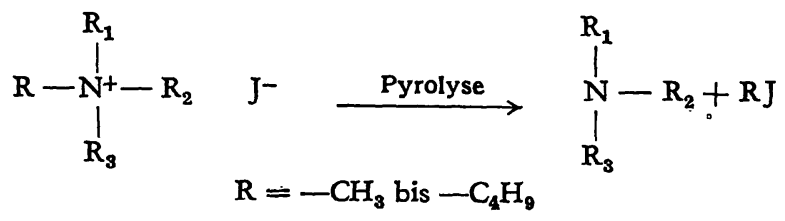

Bei der Erprobung nach den Angaben von SzILAGyi, SCHMIDT und GreEN (1) ergab sich außerdem, daß die Bestimmung des Alkylhalogenids RJ technisch wesentlich einfacher ist, reproduzierbarere Werte und eine lineare Funktion ergibt. Infolge der kürzeren Retentionszeit der Alkylhalogenide geht die Bestimmung auch schneller. Der wesentliche Nachteil unserer Methode besteht in der Notwendigkeit einer vorangehenden, möglichst einwandfreien Isolierung der $z u$ analysierenden Verbindung. Eine Abscheidung der quaternären Ammoniumverbindungen durch Komplexbildung mit Jod erleichtert die Isolierung, zumal sich das Addukt wieder spalten läßt.

\section{Material und Methoden}

\section{Gaschromatographie}

Für die Analysen wurde ein Gaschromatograph der Firma HewlettPackard, F \& M 402 verwendet.

Das Pyrolyse-Zusatzgerät mit Stromversorgungsteil ist eine Eigenkonstruktion, bei der der Pyrolyse-Aufsatz direkt auf dem Probeneinla $\beta$ befestigt wird. Eine Platinschlaufe, auf der die Lösung der Substanz aufgebracht und konzentriert wird, befindet sich im Moment der.Pyrolyse unmittelbar im Trägergasstrom innethalb des geheizten Einlaßrohres. Eine zusätzliche Trägergaszufuhr von außen ist also nicht erforderlich; ebensowenig werden Septa benötigt, die bei einigen anderen Zusatzgeräten leicht zu Undichtigkeit führen. Das kurzzeitige Erhitzen der Platinschlaufe geschieht durch einen Stromstoß, dessen Dauer und Stärke am Stromversorgungsteil eingestellt werden kann.

1 bis $5 \mu \mathrm{l}$ der wäßr. Lösungen der untersuchten Substanzeñ in einer Konzentration von mindestens $100 \mathrm{nMol} / \mathrm{ml}$ (bezogen auf die Zahl der quaternären Ammoniumgruppen im Molekül) wurden auf der Platinschlaufe des Pytolyseaufsatzes zunächst eingedampft. Anschließend wurde der Pyrolyseaufsatz mit der Trennsäule verbunden und der Rückstand im Stickstoffstrom innerhalb von 10 Sek. auf etwa $800^{\circ}$ erhitzt. 
Unsere Lösung heißt: Direkte Probenidentifikation, on-lineMeßwerterfassung und real-time-Datenverarbeitung mit dem SILAB-System. Es bringt:

oHöhere Leistung des Labors durch sinnvolleren

Einsatz der Fachkräfte bei wirtschaftlich optimaler,

Nutzung der Analysen und Meßgeräte.

oTermingerechte Analysen nach vielseitiger automatischer Kontrolle und sicherer Identifikation der Meßdaten.

- Arbeitsplatz- und patientenorientierte Zusammenstellung der automatisch verarbeiteten Daten. Ausgabe der Gesamtinformation in übersichtlichen, fehlerfreien Ergebnislisten.

Für weitere Informationen wenden Sie sich bitte an unsere nächstgelègene Geschäftsstelle oder an:

SIEMENS AKTIENGESELLSCHAFT

Med Dv · 8520 Erlangen . Postfach 400

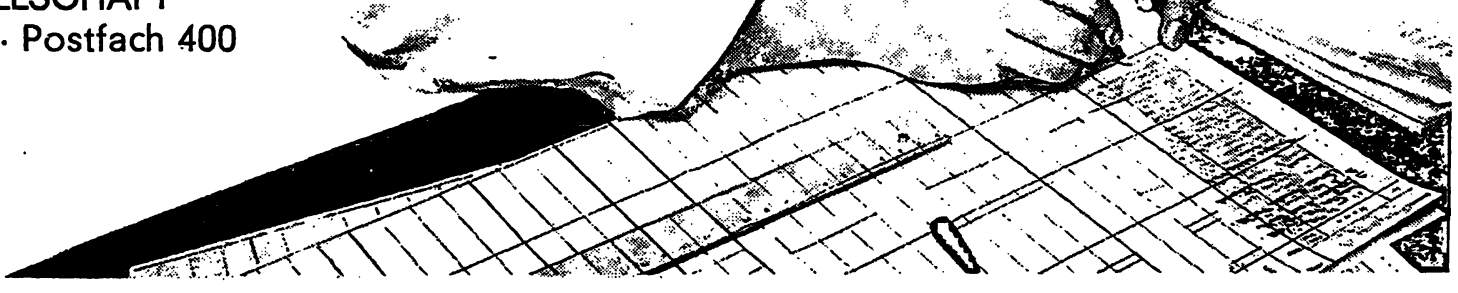

Daten automatisch erfassen wit dem SILAB-Systern von Siemens 
Wir bieten Ihnen

alle Meßgeräte zur ${ }^{3} \mathrm{H}$ - und ${ }^{14}$ C-Analyse, unter anderem den modernen Flüssigszintillationsmeßplatz Betaszint BF 5000 mit externem Kleinrechner, oder den bewährten Dünnschicht-Scanner II für ein- und zweidimensionales Scanning von Platten bis zu $200 \times 400 \mathrm{~mm}$, mit dem noch $100 \mathrm{dpm}{ }^{14} \mathrm{C}$ und $3000 \mathrm{dpm}{ }^{3} \mathrm{H}$ pro Fleck leicht gemessen werden können.

Besuchen Sie unseren Stand!
TRITIUM

in den Biowissenschaften

-Ein komplettes Geräteprogramm-

\section{Neuheit: \\ BETA KAMERA}

Wir biefen Ihnen

auch alle Peripheriegeräte von der Probenvorbereitung bis zur Datenverarbeitung. Der Verbrennungsautomat Pyroszintz. B. verarbeitet pro Tag bis zu 100 Proben mit Einzelmengen von $100 \mathrm{mg}$. Der Micro-Mat gestattet die Verbreninung direkt im LSGläschen. Dazu kommen alle für den Strahlenschutz notwendigen Meßgeräte.

Besuchen Sie unseren Stand!

\section{Amalytica 72 Halle 2 Stand 2416/2319}

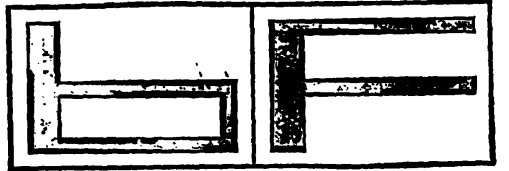

VERTRIEBS-GMBH FÜR MESSTECHNIK der Firmen Frieseke \& Hoepfner GmbH . Erlangen-Bruck und Laboratorium Prof. Dr. Rudolf Berthold - Wildbad

75 Karlsruhe 41 - Bergwaldstr. 30 - Postfach 410220 - Tel. (0721) $401011-14 \cdot$ Telex $7825927 \cdot$ Telegramm Raytec
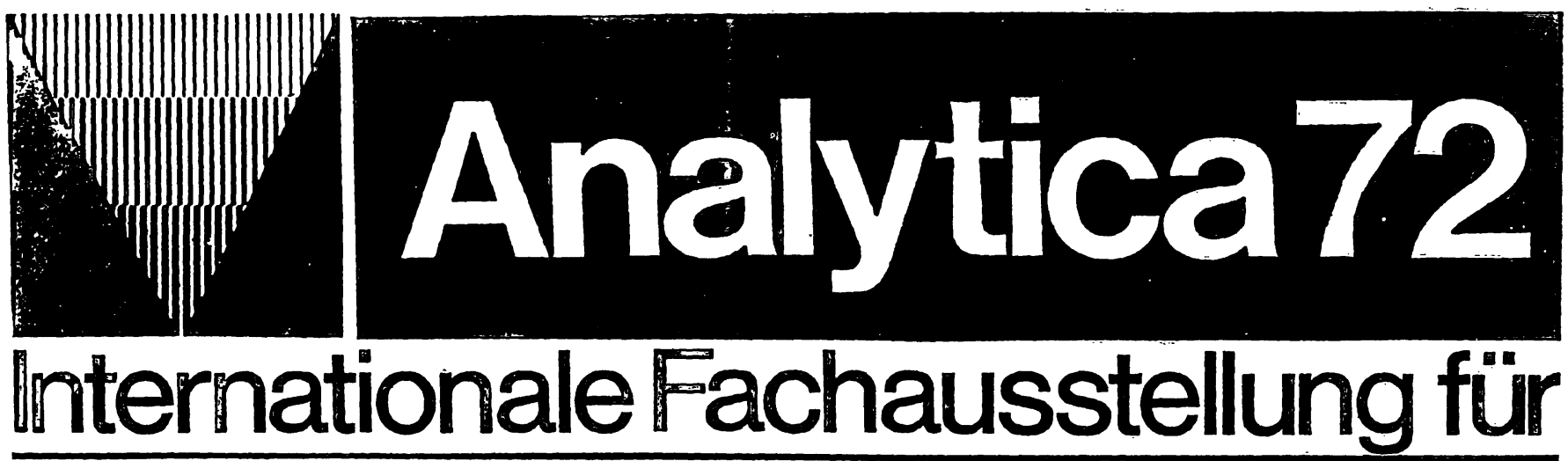

Internationale Fachausstellung für Biochemische und Instrumentelle Analysemit EuropäischerTagung: Biochemische Analytik München 25. bis 28. April 1972

Münchener Messe- u. Ausstellungsgesellschaft mbH, 8000 München 12, Postfach 200, Theresienhöhe 13, Tel. (0811) 767 11 
Wir verwendeten Glassäulen von $3 \mathrm{~m}$ Länge und etwa $3 \mathrm{~mm}$ Innendurchmesser, gefüllt mit $6 \%$ Silicone Gum Rubber SE 30 oder $10 \%$ Siliconfett auf Chromosorb G AW DMCS ( 80 bis 100 mesh). (Siliconfett: Fa. E. Merck „Siliconfett für die Gaschromatographie", sonstige flüssige und feste Phasen: Fa. HewlettPackard.) Die Ofentemperaturen betrugen: $50^{\circ}$ für Methyl- und Äthyljodid und $90^{\circ}$ für Butyljodid. Der Flammenionisationsdetektor wurde auf $120-150^{\circ}$ eingestellt. Folgende Strömungsgeschwindigkeiten erwiesen sich als zweckmäßig:

$\begin{array}{lr}\text { Trägergas }\left(\mathrm{N}_{2}\right): & 40 \mathrm{ml} / \mathrm{Min} . \\ \text { Wasserstoff: } & 30 \mathrm{ml} / \mathrm{Min} . \\ \text { synthetische Luft: } & \text { etwa } \quad 300 \mathrm{ml} / \mathrm{Min} .\end{array}$

Die Säulen wurden jeweils über Nacht bei $150^{\circ}$ ausgeheizt. Die Auswertung erfolgte nur nach der Höhe der Peaks. Sofern nämlich alle Parameter wie Trennsäule, Ofentemperatur und Strömungsgeschwindigkeit des Trägergases bei Eich- und Probenmessungen gleich sind und auch konstant gehalten werden, sind auch die Retentionszeiten, die Form und die Halbwertsbreite der Peaks gleich. Die Höhe ist dann der Fläche proportional. Die Eichkurven beweisen die Zulässigkeit dieses Vorgehens.

Bei einer Einstellung von „Range“: 1 und „Attenuator": 8 wurde eine peak-Höhe von etwa $60 \% / \mathrm{nMol}$ entstandenes Methyljodid erzielt.
Abtrennumg von Neostigmin, Succinylbisclsolin, Scopolaminbutylbromid (Buscopan), d-Tubocurarin, Metbylcurarin u. a. alls Extrakten biologischen Materials

Es können wäßr. oder alkohol. Gewebsextrakte, Blutplasma oder -serum verwendet werden, die mindestens $10 \mathrm{nMol}$ der quaternären Ammoniumverbindung enthalten sollen. Organische Lösungsmittel entfernt man und bringt auf cin Volumen von etwa $1 \mathrm{ml}$. Diese wäßr. Lösung, die auf einen pH-Wert zwischen 4 und 7 eingestellt wird, schüttelt man zweimal mit je etwa $3 \mathrm{ml}$ Chloroform. Die organische Phase wird verworfen und die wäßr. mit etwa $100 \mu \mathrm{l}$ Jod-Lösung (konzentrierte LugoL'sche Lösung aus $1 \mathrm{~g}$ Jod und $2 \mathrm{~g}$ Kaliumjodid in $20 \mathrm{~g}$ Wasser) versetzt.

Man schüttelt nun zweimal $15 \mathrm{Min}$. mit je $5 \mathrm{ml}$ Chloroform aus. (Für d-Tubocurarin hat sich Essigsäureäthylester besser bewährt.)

Danach vereinigt man die beiden organischen Phasen und versetzt mit soviel ammoniakal. Hydrazinlösung ( $2 \mathrm{ml}$ einer 24 proz. Hydrazinhydrat-Lösung werden mit $3 \mathrm{ml} 25 \mathrm{proz}$. AmmoniakLösung und $195 \mathrm{ml}$ Wasser gemischt). (200-250 $\mu \mathrm{l})$ bis die Färbung restlos verschwunden ist.

Nach $5 \mathrm{Min}$. Schütteln befinden sich dic quaternären Stickstoffverbindungen als Jodide nun wieder in der wäßr. Phase und können chromatographisch aufgetrennt oder auch direkt pyrolysiert werden.

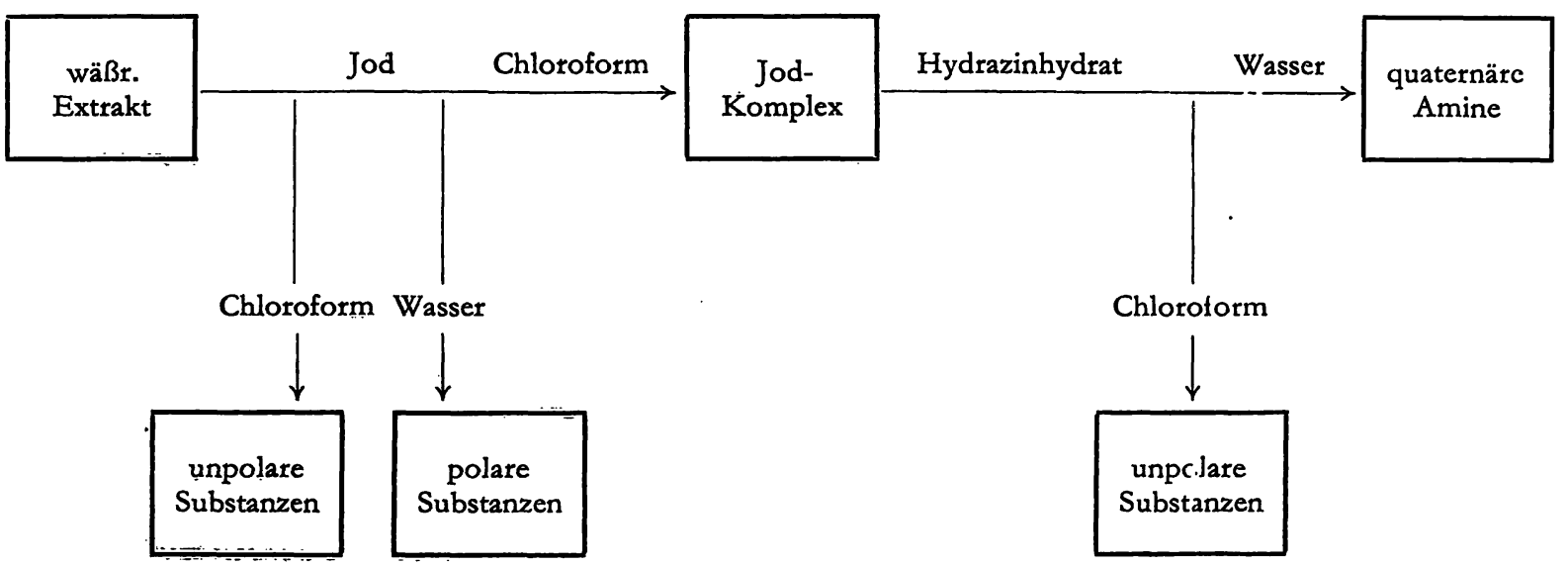

Abb. 1

\section{Ergebnisse}

Bei dẹ Pyrolyse von tetrasubstituierten Ammoniumsalzen entstehen Alkylhalogenide und tertiäre Amine oder deren weitere Zersetzungsprodukte.

Cholin und dessen Ester, Neostigmin, d-Tubocurarin, Methylcurarin u. a. liefern Methyljodid, wenn man die Jodide einsetzt.

Das Jodid von $\mathbf{N}=$ Triäthylaminoäthanol (Triäthylcholin) spaltet Äthyljodid ab und Scopolaminbutyljodid (Buscopan) hauptsächlich Butyljodid neben wenig Methyljodid. Der Beweis erfolgte durch Pyrolyse von Tetramethylammoniumjodid beziehungsweise der entsprechenden Äthyl- und Butylverbindung als Referenzsubstanzen. Diese hatten die gleichen Retentionszeiten wie die Pyrolyseprodukte der untersuchten Verbindungen (z. B. Cholinjodid, Triäthylcholinjodid oder Scopolaminbutyljodid).

Bei gleichzeitiger Arwesenheit gleicher Konzentrationen an Chlorid-, Bromid- und Jodidionen wird zu mehr als $90 \%$ das Alkyljodid gebildet. Daher wurden vor der
Pyrolyse stets Jodidionen in Form von Kaliumjodid oder besser Jodwasserstoffsäure im Überschuß zugesetzt um eindeutige Produkte zu erhalten, d. h. nur die jeweiligen Alkyljodide ohne Beimengung von Bromiden oder Chloriden.

Unter den angegebenen Bedingungen wurden folgende Retentionszeiten gemessen:

a) Bruttorecentionszeit. Sit: ergibt sich als Gesamtzeit vom Beginn des Erhitzens der Platinschlaufe bis zum Maximum des Peaks für:

Butyljodid (3): 2 Min.

Methyljodid (4): 0,9 Min.

b) Retentionszeit. (Bruttoretentionszeit - Totzeit). Als Totzeit soll hier die Zeit vom Beginn des Erhitzens bis zum Erscheinen des ersten undefinierten Peaks verstanden werden.

Es ergibt sich dann für die Retentionszeit:

Butyljodid: 1,6 Min.

Methyljodid: 0,6 Min. 
Abb. 2 und 3

Chromatogramme der Pyrolyseprodukte von in je $3 \mu$ l Lösung enthaltener Substanz

Säule: $3 \mathrm{~m}$ lang / $3 \mathrm{~mm}$ Innendurchmesser, $6 \%$ SE 30 auf Chromosorb G AW DMCS

Papier-Vorschub: $0,5 \mathrm{inch} / \mathrm{Min}$.

"1" Offnen des Säuleneinlasses

"," Beginn der Aufheizung der Platinschlaufe

"3" Peak des Butyljodids

"4" Peak des Methyljodids

Abb. 2

Lösung von $2 \mathrm{mMol} / \mathrm{l}$ Scopolaminbutylbromid (Buscopan) mit Zusatz von Jodwasserstoffsäure im Überschuß

Ofentemperatur: $90^{\circ}$

"Attenuator": 64, "Range": 1 (1/64 der maximalen Empfindlichkeit des Geräts)

Abb. 3

Lösung von $0,8 \mathrm{mMol} / \mathrm{l}$ Neostigminchlorid mit Zusatz von Jodwasser-

Ofentemperatur: $50^{\circ}$ stoffsäure im Überschuß

"Attenuator": 32, „Range“: 1 (1/32 der maximalen Empfindlichkeit)

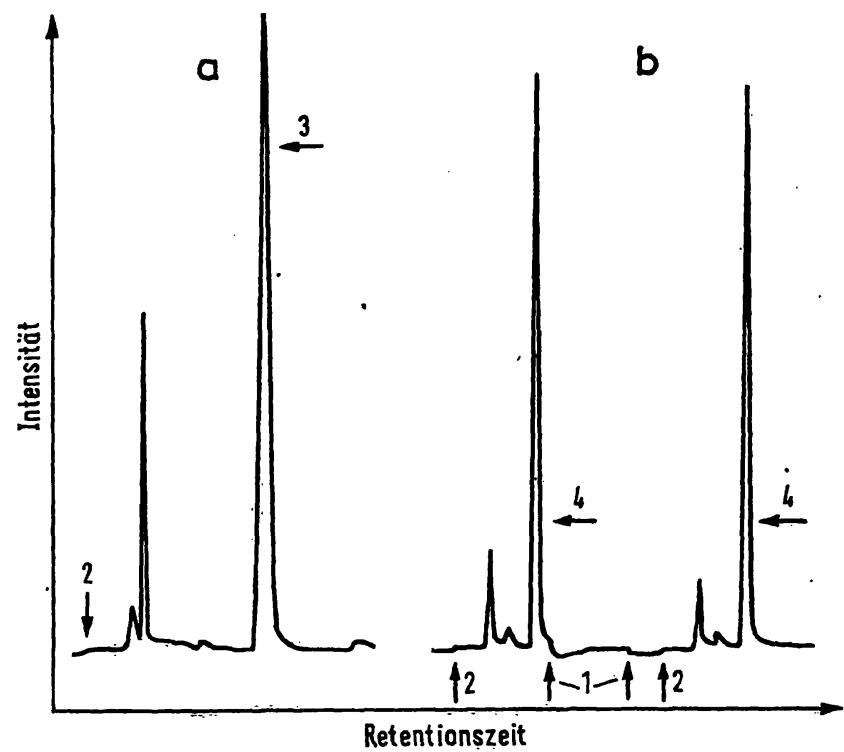

Abb. 4

Verhältnis von Menge an pyrolysiertem d-Tubocurarin und Höhe der Peaks des Methyljodids in den Bereichen von:

b) $0,1 \ldots 1,2 \mathrm{nMol}$ und a) 1,2 . $15 \mathrm{nMol}$
Die Peaks der ebenfalls entstehenden tertiären Amine treten unter diesen Bedingungen nicht in Erscheinung. Vermutlich verlassen einige Spaltprodukte die Säule erst beim Ausheizen.

Die Messungen für die Eichkurven wurden durchgeführt mit Lösungen der Konzentrationen von 0,1 bis 5,0 mMol/1 quaternären Stickstoff. Die Steigungen der Eichgeraden aller Methyljodid-abspaltenden Verbindungen sind gleich, wenn mit gleichen Einstellungen gearbeitet wird und auf quaternäre Ammoniumgruppen bezogen wird. Butyljodid wird nach Pyrolyse von Scopolaminbutyljodid (Buscupan) etwa um den Faktor 1,4 (nur auf die Peak-Höhe bezogen) höher angezeigt.

Die Abtrennung und Reinigung von Cholin und seinen Estern wird in der Regel durch papierchromatographische oder ähnliche Verfahren durchgeführt werden. Die anfallenden Eluate können, nachdem sie auf ein definiertes Volumen gebracht worden sind, direkt zur Pyrolyse gelangen. Noch besser eignen sich Eluate aus Cellulose-beschichteten Dünnschicht-Platten oder -Folien.

Für die weitgehende Vorreinigung von Neostigmin, Methylcurarin, d-Tubocurarin, Scopolaminbutyljodid (Buscopan) und mit schlechterer Ausbeute auch Suc- cinylbischolin aus wäßr. Extrakten eignet sich das Verfahren der Abtrennung in Form der Jodkomplexe (Abb. 1). Diese Addukte sind wesentlich unpolarèr als die freien Ammoniumsalze und viele ihrer Begleitstoffe und deshalb leicht auszuschütteln.

Die genannten Verbindungen gewinnt man mit folgenden Ausbeuten zurück:
d-Tubocurarin:
$95 \%$
Methylcurarin:
$95 \%$
Neostigmin:
$97 \%$

Scopolaminbutyljodid

(Buscopan): $\quad 97 \%$

Succinylbischolin: $\quad 58 \%$

Im Falle des Scopolaminbutyljodid (Buscopan) reichte die so erzielte Reinheit für die pyrolytisch-gaschromatographische Bestimmung aus.

\section{Diskussion}

Das angewandte Pyrolyse-Verfahren umgeht die Nachteile der bisher gebräuchlichen Methoden:

Die chemische Demethylierung von quaternären Ammoniumsalzen mit Natriumthiophenolat erfordert sehr exaktes Arbeiten unter sorgfältigem Ausschluß von Wasser sowie speziell gereinigte und absolut wasserfreie Reagenzien (2). 
Die quantitative gaschromatographische Bestimmung der durch Pyrolyse gebildeten tertiären Amine (1) ist bei höher molekularen Substanzen wie den Curare-Alkaloiden nicht möglich und andererseits geben niedere Amine wie Dimethylaminoathanol aus Cholin schlecht auswertbare Peaks. Bei der Chromatographie derartig polarer Substanzen kommt es zu Schweifbildung - vermutlich durch Absorption an der Trägerphase und zu einem nichtlinearen Verlauf der Eichkurve. Bei Mengen unter $1 \mu \mathrm{g}$ ist dieser Effekt sehr groß und man erhält einen starken Abfall der Empfindlichkeit.

Ubliche spektroskopische Methoden sind nicht genügend empfindlich, wie zum Beispiel die „EnneajodidMethode" (5) für Cholin und Acetylcholin, deren untere Nachweisgrenze bei $20-50 \mathrm{nMol}$ liegt. FluoreszenzMessungen wie für d-Tubocurarin (6) beschrieben, sind zwar empfindlich, jedoch nicht konstant reproduzierbar.

Als Vorteile des mitgeteilten Verfahrens sind anzuführen:

a) Eine große Zahl an bestimmbaren Substanzen: z. B. auch Phosphorylcholin und Glycerylphosphorylcholin, die sich sonst nur nach Abspaltung von Cholin bestimmen lassen, was beim Phosphorylcholin sehr schwierig und langwierig ist.

b) Relativ gute Genauigkeit $( \pm 7,5 \%)$ und Empfindlichkeit (bis 0,1 nMol quaternären Stickstoff).

c) Relativ einfache Aufarbeitung der Extrakte.

d) Kurze Retentionszeiten (1-2 Min.), deshalb sind 15-20 Bestimmungen pro Stunde möglich.

e) Niedrige Säulenofentemperatur, daher gute Grundlinie und lange Haltbarkeit der Säulen.

f) Rasche Auswertung, da nur die Peak-Höhe als Maß dient.

Ein Nachteil besteht darin, daß die Methode nicht sehr spezifisch ist. Andere quaternäre Ammoniumsalze, die Alkylgruppen am Stickstoff tragen, geben bei der Pyrolyse ebenfalls flüchtige Halogenkohlenwasserstoffe und müssen daher vorher durch chromatographische oder andere Verfahren abgetrennt werden.

\section{Literatur}

1. Szilagyi, P. I. A., D. E. Schmidt und J. P. Green, Analytic. Chem. 40, 2009 (1968). - 2. Jenden, D. J., I. Hanin und S. I. Lamb, Analytic. Chem. 40, 125 (1968). Hanin, I. und D. J. Jenden, Biochem. Pharm. 18, 837 (1969). Hammar, C. G. und I. Hanin, Nature, London 220, 915 (1968). -3. Cramner, M. F., Life Sci., Part
I 7, 955 (1968). - 4. Stavinoha, W. E. und L. C. Ryan, J. Pharmacol. exper. Therap., Baltimore 150, 231 (1965). - 5. Appleton, H. D., B. N. Ladu jr. und B. B. Levy, J. biol. Chemistry 205, 803 (1953). - 6. Cohen, E. N., J. Laborat. Clin. Med., S. Louis 61, 338 (1963).
Dr. Hans-Jörg Vidic

Schering AG, Werk Charlottenburg Dept. für Mikrobiologische Chemie 1 Berlin 10

Tegeler Weg 28/33 